\title{
BREAST CARE IN BREAST-FEEDING MOTHERS: THE BENEFIT OF THERAPEUTIC LASER APPLIED BY PHYSIOTHERAPISTS
}

\begin{abstract}
INTRODUCTION AND PURPOSE Breast-feeding is promoted worldwide as it has many health and nutritional benefits. To achieve these benefits and ensure continued breast-feeding, adequate support, education and treatment are essential. Nipple pain is one of the main reasons for the discontinuation of breast-feeding even though a wide range of treatment options are available. The purpose of this study was to determine the use of therapeutic laser in the treatment and prevention of painful and/or cracked nipples in breast-feeding mothers.
\end{abstract}

METHODS A descriptive, cross-sectional questionnaire survey was conducted

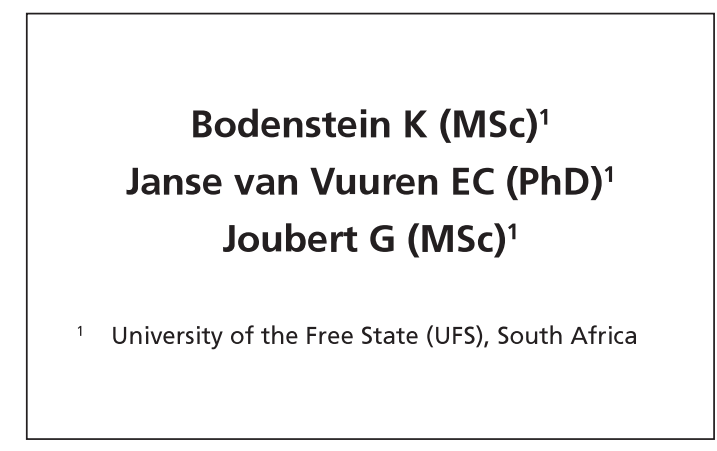
and included 101 physiotherapists. Physiotherapists working in the maternity wards in three purposively selected private hospital groups in South Africa were included.

RESULTS The response rate was $54.4 \%$. Results showed that $65 \%$ of the participants used therapeutic laser for the treatment of painful or cracked nipples, while 24\% used preventative therapeutic laser. Additionally, nipple cream was used by most patients. Referrals of patients for therapeutic laser were mostly received from the nursing staff (46.2\%) and gynaecologists (26.9\%).

DISCUSSION This study supports the early identification of nipple pain and referral for physiotherapy laser treatment. The use of therapeutic laser could therefore be included in post-natal care as it offers another comfortable and safe treatment option with no reported side effects.

KEY WORDS: BREAST-FEEDING, PHYSIOTHERAPY, LASER

\section{INTRODUCTION}

Exclusive breast-feeding of infants to six months followed by a period of continued breast-feeding up to an age of two years or older has been advocated and promoted worldwide (WHO 2007). The intended result of this nutritional plan not only has many health and nutritional benefits for the baby, mother, family and community, but also results in other important immunological, developmental, psychological, socio-economic and environmental advantages (AAP 2012, Horta et al 2007). To optimally achieve these benefits and ensure continued breast-feeding, adequate support, education and treatment need to be provided to breast-feeding mothers as it can be a challenging experience, especially for firsttime mothers (Williamson et al 2011).
A systematic review on the support for healthy breast-feeding mothers with healthy term babies revealed that extra support positively impacted on breastfeeding outcomes (Renfrew et al 2012) and should thus form an important component of health care post-partum. This was supported by Olang et al (2012) who indicated that the prevalence of exclusive breast-feeding up to six months could be increased with support and education of breast-feeding mothers. A lack of support during the early phases of breast-feeding, on the other hand, is however one of the main reasons for the early discontinuation of breast-feeding (Taveras et al 2003).

Other factors influencing the discontinuation of breast-feeding have been widely described in literature and include factors such as an insufficient milk supply, nipple pain, mastitis and engorgement of the breasts (Brand et al 2011, Morland-Schultz and Hill 2013, Page et al 2003, Vieira et al 2013). In addition to the reasons for breast-feeding discontinuation, the treatment of these factors have also been widely researched and described with special emphasis on the treatment of nipple pain and/or discomfort as a central reason for breast-feeding termination (Morland-Schultz and Hill

\section{Corresponding author:}

Bodenstein K

MSc (Physiotherapy), University of the Free State (UFS), South Africa

PO Box 1389, Bloemfontein, South Africa, 9300 +27 (51) 4013289

bodensteink@ufs.ac.za 
2013, Page et al 2003, Vieira et al 2013). Literature however contains limited reference to therapeutic laser as a treatment modality for painful nipples (Pietschnig et al 2000), even though it is applied by physiotherapists across the world and can play a valuable role in promoting successful and continued breast-feeding. Therapeutic laser provides pain relief and promotes tissue healing (Cameron 2003) which may be of value in the treatment of painful and/or cracked nipples in breast-feeding women and thus supports continued breast-feeding.

The prevalence of nipple pain, especially in the initial stages of breastfeeding, has been previously reported to be between $34 \%$ and even as high as $96 \%$, with the peak pain period within the first week of breast-feeding (Page et al 2003). A number of theories highlighted in relation to the causes for painful and/or cracked nipples during the early stages of breast-feeding included factors such as skin health, nutritional status, positioning of the infant, breast engorgement, frequency and duration of feedings, nipple anatomy and tension of the mother during feedings as possible causes (Morland-Schultz and Hill 2013, Page et al 2003, Strong 2011, Vieira et al 2013). Painful nipples can also be caused by a Staphylococcus aureus infection, usually spread from the baby's mouth to the nipple during feeding (Graves et al 2003).

Features of the nipple usually associated with nipple pain can include cracking, bleeding, swelling, erythema, white/yellow/dark stains and blisters (Morland-Schultz and Hill 2013, Vieira et al 2013). Nipple pain can however also lead to more serious complications if left untreated, as Kinlay et al (2001) reported a relation between cracked nipples and mastitis. Education with regard to the treatment of nipple pain is therefore once again highlighted, not only to ensure continued breast-feeding, but to prevent more serious complications.

Various treatments for the prevention and treatment of painful and/or cracked nipples in breast-feeding women have been described and reviewed. The most common treatment modalities include the use of warm water compresses, lanolin, hydrogel dressings, breast milk and different topical agents. The education of breast-feeding mothers on aspects such as latching and the impact thereof on nipple pain also forms an important component in the prevention and treatment of painful and/or cracked nipples (Morland-Schultz and Hill 2013, Page et al 2003, Vieira et al 2013). None of these treatment modalities however have provided the definitive solution in the treatment of nipple pain.

Pietschnig et al (2000) used therapeutic laser on patients with painful nipples as an adjunct treatment modality. This was done in combination with lanolin cream, antibiotics and antimicotics. Pain reduction from an average intensity of 9.17 out of ten to 1.95 was obtained and resulted in 28 of the 31 participating women persisting with breast-feeding. Besides positive results with regard to pain relief, Kovalev (as cited in Amir et al 2004) reported on the reduction of mastitis from $18.6 \%$ to $3.7 \%$ in 329 breast-feeding women treated with therapeutic laser for cracked nipples. These positive results highlighted the possibility that therapeutic laser could provide another safe treatment modality in the treatment of painful and/or cracked nipples as well as the prevention of mastitis.
The purpose of this study was to determine the use of therapeutic laser in the treatment and prevention of painful and/or cracked nipples in breast-feeding mothers.

\section{METHODOLOGY Study design}

A descriptive, cross-sectional questionnaire survey was conducted to determine the use of therapeutic laser in the treatment and prevention of painful and/or cracked nipples of breast-feeding women.

\section{Participant sampling and recruitment}

All physiotherapists working in the maternity wards of all hospitals in the three purposively selected private hospital groups in South Africa were included in the main study. The nursing professional in charge of the maternity ward in each hospital was contacted telephonically in order to obtain details regarding all physiotherapists working in the respective maternity wards. Initially 115 South African physiotherapists were included in the study of which 14 did not meet the inclusion criteria, thus resulting in a study sample of 101 physiotherapists.

\section{Questionnaire as outcome measure}

The questionnaire was compiled from information gathered during the extensive literature review pertaining to therapeutic laser and specifically the use thereof for pain relief and wound healing, breast-feeding as well as painful and cracked nipples (Cameron 2003, Hopkins et al 2004, Pietschnig et al 2000, Robertson et al 2006). This was further supplemented by personal interviews with physiotherapists in the field of therapeutic laser.

Table 1: Physiotherapists' perceptions on the use of therapeutic laser for painful and/or cracked nipples $(n=26)$

\begin{tabular}{|c|c|c|}
\hline QUESTION & $\begin{array}{l}\% \\
\text { YES }\end{array}$ & $\begin{array}{c}\% \\
\text { NO }\end{array}$ \\
\hline Do women receive laser therapy for painful/cracked nipples, only to stop breast-feeding anyway? & 30.8 & 69.2 \\
\hline $\begin{array}{l}\text { Do women with painful/cracked nipples report that laser therapy helped them to continue } \\
\text { breast-feeding? }\end{array}$ & 100 & 0 \\
\hline Do women with painful/cracked nipples who receive laser therapy report that it is effective? & 100 & 0 \\
\hline $\begin{array}{l}\text { Did any of your patients who received laser therapy for painful/cracked nipples experience any } \\
\text { side effects? }\end{array}$ & 0 & 100 \\
\hline
\end{tabular}


The questionnaire included seven sections containing questions on demographic information, therapeutic laser type use, therapeutic laser dosage and treatment specifics, other treatment modalities included in breast care as well as opinions on the effectiveness of therapeutic laser in the treatment of painful and cracked nipples.

\section{Pilot study}

The questionnaire was pre-tested during a pilot study and sent to ten physiotherapists: three working in the public sector, five from the private sector and two lecturers from the UFS. The pilot study tested the proposed study procedure as well as the appropriateness and comprehensibility of the questions. Participants participating in the pilot study also made suggestions on any gaps in the questionnaire. Minor changes were then made to the questionnaire and study procedure to improve the clarity of the questions and the effective execution of the data collection. As changes were made after the pilot study, data was not included in the main study.

\section{Study procedure}

Each physiotherapist was contacted telephonically and the purpose of the study briefly explained. The information document, letter of consent and questionnaire were then faxed or e-mailed in Afrikaans or English (as requested during the telephonic conversation) to all potential participants. Participants signed informed consent forms to participate in this study and anonymity was ensured by coding all data and no names or other personal identifiers were retained. Participants were requested to return the completed forms within two weeks. Participants who did not meet this deadline were sent a reminder and afforded another two weeks for submission. A third reminder was sent after the second deadline, once again containing the informed consent form as well as the questionnaire. After this two week period, participants were contacted telephonically to ensure that they received the questionnaire and were requested to submit the questionnaire as soon as possible if they agreed to participate. In a last effort to improve the response rate, a final reminder was sent to all participants reminding them of the final submission date for the questionnaire. This study was approved by the Ethics committee of the Faculty of Health Sciences at the University of the Free State (UFS), South Africa (ETOVS number 190/06) before commencement.

\section{Data analysis}

Data were analysed by the Department of Biostatistics at the UFS. Results were summarised by using frequencies and percentages and reported on by means of tables and graphs.

\section{RESULTS}

A response rate of $54.4 \%$ was achieved in this study and the 55 participants were representative of all geographical areas within South Africa. The majority of the participants were female $(87.2 \%)$, with ages ranging between 31 and 40 years $(40.4 \%)$, and had been working as physio-therapists for 11-20 years (36.2\%).

Therapeutic laser therapy for the treatment of breast-feeding women's nipples was used by 26 participants $(55.3 \%)$ and only the data from these participants were included in the reporting on laser for painful and cracked nipples. Results for therapeutic laser therapy were divided into three main categories and included therapy for painful nipples (before nipples show abrasions/cracks), therapy for cracked nipples (nipples with abrasions/ cracks) and preventative therapy (before nipples become painful or cracked).

\section{Laser therapy for painful nipples}

Most of the 26 respondents $(80 \%)$ indicated that they treated painful nipples with therapeutic laser, whilst $75 \%$ treated the nipples before abrasions/ cracks appear. Treatment for painful nipples was usually performed on a daily basis $(50 \%)$, either in contact with the skin $(45 \%)$ or at a distance of less than one centimeter from the skin $(55 \%)$. As with preventative laser therapy, participants again indicated that therapeutic laser therapy for painful nipples reduced the occurrence of cracked nipples $(85 \%)$.

\section{Laser therapy for cracked nipples}

A quarter of the respondents (25\%) only started treatment with therapeutic laser once the breast-feeding women's nipples had abrasions/cracks. In these cases, treatment was given daily and twice daily during hospitalisation by an equal number of participants $(34.8 \%)$.

When treating cracked nipples, most treated the whole nipple with increased application over the area with the lesion (39.1\%), while $34.8 \%$ treated only the area with the lesion. Treatment was again given either in contact with the skin $(39.1 \%)$ or at a minimal distance $(60.9 \%)$.

\section{Preventative laser therapy}

Preventative laser therapy was applied by $24 \%$ of the respondents, implying that they initiate treatment before the nipples become painful or cracked. Those using therapeutic laser therapy preventatively mainly treat the nipples on a daily basis $(66.6 \%)$ and the majority $(83.3 \%)$ indicated that preventative therapeutic laser therapy prevented painful or cracked nipples and thus supported continued breast-feeding.

\section{Other treatment modalities}

Thirteen of the respondents using laser therapy indicated that their patients did not exclusively receive laser therapy. A supplementary nipple cream was the most frequently added modality used by $84.6 \%$ of the participants' patients when the nipple was painful or cracked.

\section{Support and referral for therapeutic laser therapy}

Physiotherapists who participated in this study support the use of therapeutic laser for the treatment of painful and/or cracked nipples in breast-feeding women as indicated by the results in Table 1 below.

Support for therapeutic laser therapy is corroborated by the referral of patients with painful and cracked nipples by the nursing staff in the participating hospitals' maternity wards $(46.2 \%)$. The survey further indicated that $84.6 \%$ of participants felt that gynecologists were aware of laser therapy for painful and/ or cracked nipples, even though only $26.9 \%$ indicated that they received referrals from the gynaecologist.

\section{Discussion and Clinical Implications}

Nipple pain associated with breast-feeding 
is a common problem and often results in the early discontinuation of breast-feeding. A number of treatment modalities have been described to address this problem, although not one has been proven superior to another (Morland-Schultz and Hill 2013, Page et al 2003, Vieira et al 2013).

Therapeutic laser is often used by physiotherapists for the treatment of painful and/or cracked nipples associated with breast-feeding and offers another comfortable and safe treatment option with no reported side effects. Positive results in the treatment of nipple pain and mastitis using therapeutic laser have been described in literature (Kovalev as cited in Amir et al 2004, Pietschnig et al 2000) and the use thereof by physiotherapists in South Africa has been confirmed by this study $(55.3 \%)$. This study also included the use of therapeutic laser in preventative care $(23.1 \%)$, which has not been described in the literature before.

The respondents in this study indicated that preventative laser treatments reduce the occurrence of painful and/or cracked nipples in breast-feeding mothers, and that laser therapy for patients with painful nipples reduces the occurrence of cracked nipples. As painful and/ or cracked nipples have been identified as one of the main reasons for the early discontinuation of breast-feeding (Brand et al 2011), these results have an important clinical implication.

The effective utilisation of therapeutic laser as a treatment modality is however dependant on the referral and cooperation of all professionals involved in the pre- and post-natal care of breast-feeding women. Participants in this survey indicated that patients with painful and/or cracked nipples were mostly referred for therapeutic laser therapy by the nursing staff in the maternity ward $(46.2 \%)$, even though most participants $(84.6 \%)$ were of the opinion that gynaecologists were also aware of this treatment option. It is therefore important that all professionals involved in post-partum care are educated on all available treatment options for painful and/or cracked nipples to ensure that they can educate and/or support breast-feeding mothers appropriately and effectively. Besides continuation of breast-feeding, a more positive feeding experience could also support a more healthy relationship between the mother and infant and positively influence the mothers' psychological well-being (Williamson et al 2011).

An added advantage of therapeutic laser treatment is that it is not time consuming and the breast-feeding mother could thus be further supported whilst adapting to her new circumstances and routine, without infringing on her time. Treatment times for the treatment of painful and/or cracked nipples can range between 18 to 33 seconds per $\mathrm{cm}^{2}$, depending on the type of laser used and intended laser dosage (Cameron 2003). As the intensity of nipple pain usually peaks within the first two weeks postpartum (Strong 2011), it is important that professionals involved in early postpartum care (especially nursing staff or midwives) identify and refer patients in need of treatment for painful and/or cracked nipples. The therapeutic laser apparatus is small, portable and often battery-operated ensuring that it can be easily transported and is thus suitable for in-hospital treatments or home visits (Cameron 2003).

Therapeutic laser treatment can also be given at a distance from the skin, thus eliminating or limiting skin contact. This has two advantages in the treatment of painful and/or cracked nipples, namely limiting treatment pain (due to contact), and also reducing the risk of infection. Infection associated with cracked nipples and the use of nipple creams on cracked nipples leading to mastitis, is described by Kinlay et al (2001). Mastitis leads to breast-feeding cessation and the reduction or prevention thereof by means of therapeutic laser therapy may well be clinically important for the continuation of breast-feeding (Kovalev as cited in Amir et al 2004).

\section{Limitations and Recommendations}

Potential participants in the public sector were excluded. This study shows interesting trends, but the study sample was small and over or underreporting could be a limitation. The preventative laser treatment of nipples, that is treatment before the nipples become painful or cracked, is not described in literature.

A follow-up clinical trial to test the efficacy of this treatment could be under- taken. A clinical trial could also more effectively describe the laser dosages and treatment specifics for the treatment of painful and cracked nipples.

\section{CONCLUSION}

Many treatments for painful and cracked nipples, including therapeutic laser treatments, have been studied. None of these treatments, however, have been shown superior to another and a solution for early cessation of breastfeeding as a result of painful and/or cracked nipples has not been found.

This study supports the early identification of nipple pain and referral for physiotherapy laser treatment which appears helpful for relieving the symptoms that often cause early cessation of breastfeeding. The continuation of breast-feeding could thus be supported by the use of therapeutic laser therapy to reduce painful and/or cracked nipples and could therefore be included in therapeutic, educational and supportive initiatives associated with pregnancy and post-natal care. Therapeutic laser is thus a safe, effective, adjuvant treatment in the treatment of painful and cracked nipples during breast-feeding.

\section{REFERENCES}

AAP (American Academy of Pediatrics) 2012 Breastfeeding and the use of human milk. Policy statement. Pediatrics 129(3):e827-e841

Amir LH, Lumley J, Garland SM 2004 A failed RCT to determine if antibiotics prevent mastitis: Cracked nipples colonized with Staphylococcus aureus: A randomized treatment trial. BMC Pregnancy and childbirth 4(19):1-6 of 6 [Online]. Available: http:// www.biomedcentral.com [6 September 2006]

Brand E, Kothari C, Stark MA 2011 Factors related to breastfeeding discontinuation between hospital discharge and 2 weeks postpartum. The Journal of Perinatal Education 20(1):36-44

Cameron MH 2003 Physical Agents in Rehabilitation. From Research to Practice. Saunders, St. Louis

Graves S, Wright W, Harman R, Bailey S 2003 Painful nipples in nursing mothers: Fungal or staphylococcal? Australian Family Physician 32(7):570-571 
Hopkins JT, McLoda TA, Seegmiller JG, Baxter GD 2004 Low-level laser therapy facilitates superficial wound healing in humans: A tripleblind, sham-controlled study. Journal of Athletic Training 39(3):223-229

Horta BL, Bahl R, Martines JC, Victora CG 2007 Evidence on long-term effects of breastfeeding. Systematic reviews and meta-analyses. Department of Child and Adolescent Health and Development (CAH), World Health Organization, Switzerland

Kinlay JR, O'Connell DL, Kinlay S 2001 Risk factors for mastitis in breastfeeding women: Results of a prospective cohort study. Australian and New Zealand Journal of Public Health 25(2):115-120

Morland-Schultz K, Hill PD 2005 Prevention of and therapies for nipple pain: A systematic review. Journal of Obstetric, Gynaecologic and Neonatal Nursing 34(4):428-437 [Online]. Available: http:// www.blackwellsynergy.com/doi/pdf/10.1177/ 0884217505276056- [3 June 2013]
Olang B, Heidarzadeh A, Strandvik B, Yngve A 2012 Reasons given by mothers for discontinuing breastfeeding in Iran. International Breastfeeding Journal 7:1-7 of 7

Page T, Lockwood C, Guest K 2003 Management of nipple pain and/or trauma associated with breast-feeding. Johanna Briggs Institute (JBI) Reports 1:127-147

Pietschnig B, Pani M, Käfer A, Bauer WE, Lischka A 2000 Use of soft laser in the therapy of sore nipples in breastfeeding women. Advances in experimental medicine and biology 478:437-438

Renfrew MJ, McCormick FM, Wade A, Quinn B, Dowswell T 2012 Support for healthy breastfeeding mothers with healthy term babies The Cochrane Collaboration: Wiley and Sons, Ltd., United Kingdom

Robertson V, Ward A, Low J, Reed A 2006 Electrotherapy explained. Principles and Practice. Butterworth Heinemann Elsevier, Edinburgh
Strong GD 2011 Provider management and support for breastfeeding pain. Journal of Obstetric, Gynecologic and Neonatal Nursing 40:753-764

Taveras EM, Capra AM, Braveman PA, Jensvold NG, Escobar GJ, Lieu TA 2003 Clinician support and psychosocial risk factors associated with breastfeeding discontinuation. Pediatrics 112(1):108-115

Vieira F, Bachion MM, Mota DDCF, Munari DB 2013 A systematic review of the interventions for nipple trauma in breastfeeding mothers. Journal of Nursing Scholarship 45(2):1-9

WHO (World Health Organization) 2007 Infant and young child feeding. Model chapter for text books for medical students and allied health professionals. WHO Press, Switzerland

Williamson I, Leeming D, Lyttle S, Johnson S 2011 'It should be the most natural thing in the world': exploring first time mothers' breastfeeding difficulties in the UK using audio-diaries and interviews. Maternal and Child Nutrition 8:434-447 